\title{
Digital MEMS Microphone with Inter-IC Sound Interface for Photoacoustic Spectroscopy
}

\author{
Henry Bruhns, André Marianovich, Stephan Rhein, Marcus Wolff \\ Hamburg University of Applied Sciences, Institute for Physical Sensors, Berliner Tor 21, \\ 20099 Hamburg \\ henry.bruhns@haw-hamburg.de
}

\begin{abstract}
In Photoacoustic Spectroscopy that is adopted for gas sensing, standard condenser microphones are usually used to detect the pressure variation inside of the sample cell. We present the application of a new commercial $I^{2} S$ digital MEMS microphone that is enhanced with a filter and an analog to digital converter in a single package. The utilization of the described MEMS microphone together with an embedded microcontroller would significantly reduce the required space and costs for the components needed to realize the signal detection path of the spectrometer. We present an initial estimation of the improvements regarding sensitivity, signal-to-noise ratio and dynamic range on the base of the MEMS microphone's data sheet compared with the conventional signal path with a capacitive microphone, a microphone preamplifier and a lock-in amplifier.
\end{abstract}

Key words: Photoacoustic spectroscopy, MEMS microphone, condenser microphone

\section{Photoacoustic spectroscopy}

The basic principle of photoacoustic spectroscopy is the usage of the transformation of absorbed modulated radiation into an acoustic wave that is then detected with a microphone and phase-sensitively amplified [1]. The quality of the used microphone and all other components of the signal path from the microphone to the phase-sensitive amplifier clearly influences the sensitivity of the spectroscope. Two different designs of the signal path will be discussed: The standard design with conventional condenser microphone and a new design with digital MEMS microphone.

\section{Comparison of Designs}

The overall signal path of the standard setup consists of the following cascaded two-ports: Condenser microphone, cable connection between microphone and microphone preamplifier, microphone preamplifier, cable connection between microphone preamplifier and lock-in amplifier, input filter and input amplifier of the lock-in amplifier. The signal processing of the used lock-in amplifier is performed by a digital signal processor. The input signal is converted with the main analog to digital converter (ADC) which is placed directly after the input amplifier. The input to the main ADC can directly be monitored. Its resolution is 18 bit. The digital output of this ADC is inaccessible to the user, but it can be observed that the lock-in amplifier accepts input signals that produce $10 \mathrm{~V}$ at the monitor output without triggering the overlload indicators of the device. Therefore, it can be assumed that the main ADC's full-scale range is matched to this value.

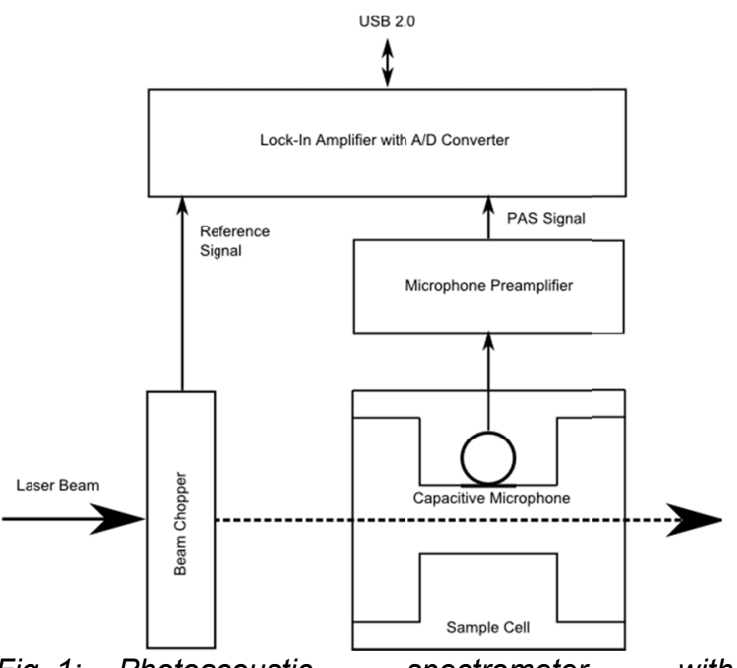

Fig. 1: Photoacoustic spectrometer with conventional signal processing path

In the new setup the condenser microphone, together with its analog signal path, up to the lock-in amplifier, is replaced by a sole digital MEMS microphone. The signal processing function of the lock-in amplifier itself is replaced by an embedded microcontroller which is directly connected to the digital interface of the digital MEMS microphone. 


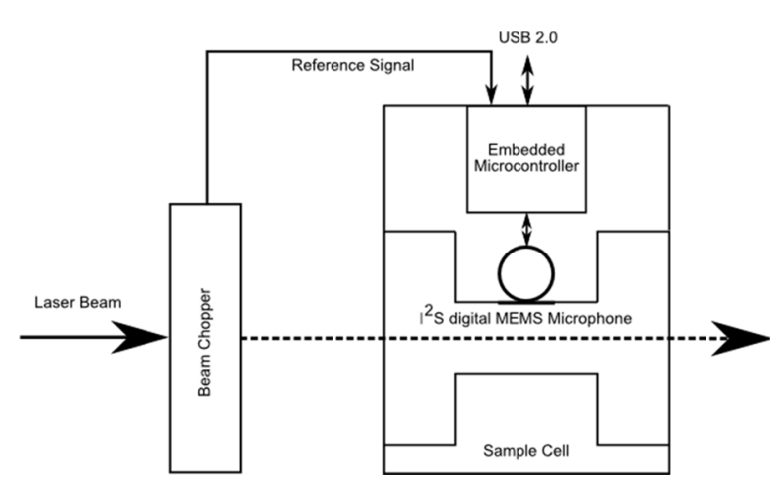

Fig. 2: Photoacoustic spectrometer with signal processing path based on new $I^{2} S$ digital MEMS microphone and embedded microcontroller

\section{Estimating the Improvements of the new Method}

With respect to the properties of communication systems, three quantities will be determined: Sensitivity, signal-to-noise ratio, and dynamic range. The terms will be defined and the differences between the standard and the MEMS microphone based solution will be elucidated.

\subsection{Sensitivity}

The sensitivity of a microphone is the electrical response at its output to a given standard acoustic input. The acoustic input is a sine wave with a frequency of $1 \mathrm{kHz}$ and a sound pressure level (SPL) of $1 \mathrm{~Pa}$. This value is usually referred to as the standard sound pressure level (standard SPL). If this value is compared to the sound pressure level at the threshold of human audibility, which is at a sound pressure level of $20 \mu \mathrm{Pa}$ and expressed in decibel, the standard sound pressure level is $94 \mathrm{~dB}$. For analog microphones the sensitivity is typically expressed in decibel with respect to a reference output voltage of 1 Volt [2].

$$
S_{a b V}=20 \log _{10} \frac{S_{m v} / P a}{1 V / P a}
$$

In the case of digital MEMS microphones no output voltage can be measured. Therefore, the sensitivity is expressed as the percentage of the full-scale digital output that is generated by the standard sound pressure level of $1 \mathrm{~Pa}$ at 1 $\mathrm{kHz}[1]$.

$$
S_{d b F S}=20 \log _{10} \frac{S_{\% F S}}{100}
$$

Consequently, for a direct comparison of the analog and the digital solution the output value of the lock-in amplifier's ADC must be observed while the microphone is stimulated with the standard SPL of $94 \mathrm{~dB}$ at $1 \mathrm{kHz}$. This measurement was taken under the following conditions: The input limit of the microphone preamplifier was set to $1.25 \mathrm{mV}$ and the input limit of the lock-in amplifier was set to $1 \mathrm{~V}$. The lock-in amplifier's gain was set to $10 \mathrm{~dB}$. At the monitor output $5.85 \mathrm{~V}$ could be measured, which resulted in a sensitivity of $15 \mathrm{~dB}$ related to the reference voltage of $1 \mathrm{~V}$. With respect to the assumed full-scale range of the $A D C$ the sensitivity will be approximately $-6 \mathrm{dBFS}$. The sensitivity of the MEMS microphone is -29 to -23 dBFS

\subsection{Signal to Noise Ratio (SNR)}

The signal-to-noise ratio of an analog microphone is the difference in decibel between the noise level and the standard SPL.

$$
S N R_{m i c}=20 \log _{10} \frac{S P L_{\text {noise }}}{S P L_{\text {standard }}}
$$

If this value is to be estimated for the given analog signal path, another measurement has to be performed: The lock-in amplifier's ADC output must be observed whereas the microphone is not stimulated by any sound pressure level. A primary estimation can be made if the cascade of the two-ports along the whole analog signal path is surveyed with respect to the Friis Formula [3].

A component's noise figure is defined as the factor of its input to output signal-to-noise ratios.

$$
N f=\frac{S N R_{\text {input }}}{S N R_{\text {output }}}
$$

The microphone preamplifier's noise figure can be calculated as the factor of the microphone's signal-to-noise ratio and the preamplifier's output signal-to-noise ratio.

$$
N f_{\text {preamp }}=\frac{S N R_{\text {mic }}}{S N R_{\text {preamp out }}}
$$

The lock-in amplifier's noise figure will be a factor of the preamplifier's output signal-tonoise ratio and the lock-in amplifier's output SNR. 


$$
N f_{\text {lockinamp }}=\frac{S N R_{\text {preamp out }}}{S N R_{\text {lockinamp out }}}
$$

According to the Friis Formula, the total noise figure will be:

$$
N f_{\text {total }}=N f_{\text {preamp }}+\frac{N f_{\text {lockinamp }}-1}{g_{\text {preamp }}}
$$

With $g_{\text {preamp }}$ being the microphone preamplifier's gain. From eq. (7), it can be seen that the noise figure of the microphone preamplifier has a significant influence on the total noise figure of the cascaded system [3]. As the noise of the "quiet environment" cannot be defined for an exact frequency, like the signal of the standard sound pressure level, a filter is used for the measurements. This filter reduces the signal bandwidth to reflect the sensitivity of the human ear. The resulting measurements are described as "A-weighted". If the results are expressed in decibel in this case, the unit dBA is used. When this measurement was taken from the monitor output of the lock-in amplifier, a signal-to-noise ratio of approximately $40 \mathrm{dBA}$ was calculated. The signal-to-noise ratio of the MEMS microphone is $61 \mathrm{dBA}$.

\subsection{Dynamic Range}

The dynamic range is the difference between the noise floor and the maximum output level to which the microphone still responds linearly. For the digital MEMS microphone this value can be taken from the data sheet [4]. In the case of the given analog system the total dynamic range cannot easily be investigated as a result of a component mismatch. The maximum input range of the used microphone preamplifier is not high enough to avoid an overload. If the input range is reduced, the gain of an amplifier will increase. This will lead to higher sensitivity, a lower dynamic range and possibly to a worse signal-to-noise ratio.

At the given setup (input limit of the microphone preamplifier set to $1.25 \mathrm{mV}$ and input limit of the lock-in amplifier set to $1 \mathrm{~V}$ ) the output voltage of the microphone preamplifier is $500 \mathrm{mV}$ at $94 \mathrm{~dB}$ SPL. To avoid clipping of the lock-in-amplifier the sound pressure level could be increased to $100 \mathrm{~dB}$ as the maximum sound pressure level $\left(S P L_{\text {max }}\right)$. The dynamic range can be calculated by subtraction of the equivalent input noise (EIN) of the signal path from the maximum sound pressure level. The equivalent input noise is expressed as the theoretical external SPL placed at the microphones input that corresponds to the output noise floor of the signal path.

$$
E I N=94 d B-S N R
$$

The dynamic range is calculated to:

$$
\text { Dynamic Range }=S P L_{\max }-E I N
$$

The dynamic range of the used standard signal path reduces to $46 \mathrm{~dB}$. The dynamic range of the MEMS microphone is $87 \mathrm{~dB}$.

\section{Conclusion}

Table 1 shows the comparison between the measured values of the conventional system and the values of a digital MEMS microphone [4]. The results of this initial estimation suggests that the usage of a digital MEMS microphone will considerably increase the signal-to-noise ratio and the dynamic range of the signal path.

Table 1: Results of the initial estimation

\begin{tabular}{|c|c|c|}
\hline & $\begin{array}{c}\text { Conventional } \\
\text { Signal Path }\end{array}$ & $\begin{array}{c}\text { MEMS } \\
\text { Microphone } \\
\text { Signal Path }\end{array}$ \\
\hline $\begin{array}{c}\text { Sensitivity } \\
\text { in dBFS }\end{array}$ & -6 & -29 to -23 \\
\hline $\begin{array}{c}\text { Signal-to- } \\
\text { Noise Ratio } \\
\text { in dBA }\end{array}$ & 40 & 61 \\
\hline $\begin{array}{c}\text { Dynamic } \\
\text { Range in } \\
\text { dB }\end{array}$ & 46 & 87 \\
\hline
\end{tabular}

The described concept will be implemented and the results will be published in a forthcoming paper.

\section{References}

[1] M. Wolff et al., Photoacoustic detection of volatile organic compounds, SPIE, 8073, 8073A-37 (2011)

[2] J. Lewis, Microphone Specifications Explained, AN-1112, Analog Devices (2011)

[3] A. B. Carlson, et al., Communication Systems: An Introduction to Signals and Noise in Electrical Communication, Fourth Edition, McGraw-Hill (2002)

[4] ADMP411 Data Sheet Rev. 0, Analog Devices (2011) 